\title{
Observations of PSR B1259-63/LS 2883 around the 2014 periastron passage with SALT
}

\author{
Brian van Soelen*广 \\ University of the Free State, Nelson Mandela Drive, Bloemfontein, 9301, South Africa \\ E-mail: vansoelendduts.ac.za
}

\section{P. Väisänen}

South African Astronomical Observatory, PO Box 9 Observatory, 7935, South Africa; Southern

African Large Telescope, PO Box 9 Observatory, 7935, South Africa

E-mail: petriesaao.ac.za

\section{A. Odendaal, L. Klindt, P.J. Meintjes,}

University of the Free State, Nelson Mandela Drive, Bloemfontein, 9301, South Africa

E-mail: WinkAdufs.ac.za, lizelkeklindtegmail.com,

MeintjPJdufs.ac.za

\section{Sushch}

Centre for Space Research, North-West University, Potchefstroom, 2520, South Africa

Astronomical Observatory of Ivan Franko National University of L'viv, vul. Kyryla i Methodia,

8, 79005, L'viv, Ukraine

E-mail: vusushchegmail.com

The gamma-ray binary system PSR B1259-63 went through periastron on the 4th of May 2014. Approximately 17 days before periastron the pulsar passes through or near the extended circumstellar disc of the Be companion star, as evidenced by the pulsar eclipse. The close interaction is expected to disturb the disc during this period. Approximately 30 days after the previous periastron passage, in 2010, an unusual flare event was observed by Fermi-LAT which showed no clear evidence of a corresponding multi-wavelength flare in e.g. optical, X-ray or TeV gammaray. During 2014 the system was the focus of multi-wavelength campaigns to monitor the system from radio to $\mathrm{TeV}$ gamma-ray energies. We report on optical spectroscopy undertaken with the Southern African Large Telescopes from 33 days before until 78 days after the 2014 periastron passage, focusing on the $\mathrm{H} \alpha$ and He-I emission lines. The lines, which originate from the circumstellar disc, present an orbital modulation, with an increase in their equivalent widths, which peaks after periastron, and an associated change in the V/R ratio of the He-I line. The variation of the lines is similar to what was previously reported around the 2010 periastron.

SALT Science Conference 2015 -SSC2015-

1-5 June, 2015

Stellenbosch Institute of Advanced Study, South Africa

\footnotetext{
* Speaker.

${ }^{\dagger}$ Based, on observations made with the Southern African Large Telescope (SALT) under programs 2013-02-RSA003 and 2014-01-RSA-001 (PI: B. van Soelen)
} 


\section{Introduction}

The gamma-ray binary system PSR B1259-63/LS 2883, consisting of a $48 \mathrm{~ms}$ pulsar in an approximately 3.4 year orbit around the Be star LS 2883 [四, last went through periastron on the 4th of May 2014. A shock forms in the system between the pulsar and stellar winds and within this shock, particle acceleration is believed to occur, leading to non-thermal radio, X-ray and gammaray emission. This emission is brightest around periastron, with indications of maxima slightly before and slightly after periastron. These have been suggested to correspond to the periods when the pulsar passes through the circumstellar disc surrounding LS 2883 (see Chernyakova et al. [వ] and references therein for a discussion of the 2010 periastron observations). While there is a reasonable consistency between the radio, $\mathrm{X}$-ray and $\mathrm{TeV}$ gamma-ray observations, the FermiLAT GeV observations, observable for the first time in 2010/2011, showed an unusual flare starting approximately 30 days after periastron [B]. This Fermi flare occurred at a time when the emission at other wavelengths was already beginning to decrease. This lead to a number of multi-wavelength campaigns of PSR B1259-63 around the 2014 periastron passage. The source has subsequently been re-detected at $\mathrm{GeV}$ gamma-rays in 2014, at approximately the same orbital phase [ $\mathrm{H}$, 国].

A number of models have been investigated to explain the $\mathrm{GeV}$ component but there is cur-

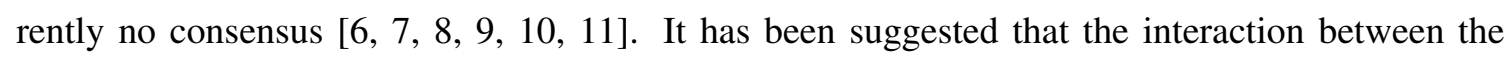
pulsar and disc near the second disc crossing could have an important effect on the gamma-ray emission and it is therefore important to follow the variation in the circumstellar disc around periastron. Previous observations showed a variation in the disc associated $\mathrm{H} \alpha$ and $\mathrm{He} \mathrm{I}(6678 \AA$ ) lines but there were no observations around the time of the Fermi flare [ $[$ ] $]$. Here we report on optical spectroscopy undertaken with the Robert Stobie Spectrograph (RSS) to monitor these lines around the 2014 periastron passage.

\section{Observations}

PSR B1259-63/LS 2883 was observed 25 times between 30 April 2014 and 21 July 2014, using the RSS (33 days before, until 78 days after periastron). The spectrograph was configured for a wavelength coverage of 6177-6983 $\AA$, with a resolving power at the central wavelength of $\mathrm{R}=11000$. Each observation consisted of $\approx 4$ exposures of approximately 500 seconds. Unfortunately poor weather and technical problems hampered observations near the onset of the Fermi flare.

\section{Data reduction and analysis}

Individual exposures were combined to form a single averaged spectra and data reduction followed the standard IRAF procedures. The shape of both lines changes around periastron, as shown in Fig. 1. The equivalent width of the $\mathrm{H} \alpha$ line was measured by integrating over the line with the standard IRAF procedures. Since the He I line always lies within an absorption feature, its equivalent width has been measured by fitting two Gaussian profiles to the violet $(\mathrm{V})$ and red (R) components, relative to the continuum, and determining the integrated area of the fits. The Gaussian profiles were also used to measure the V/R variation in the line structure. The statistical 
error in the equivalent width has been determined following the method outlined in Vollmann \& Eversberg [12].
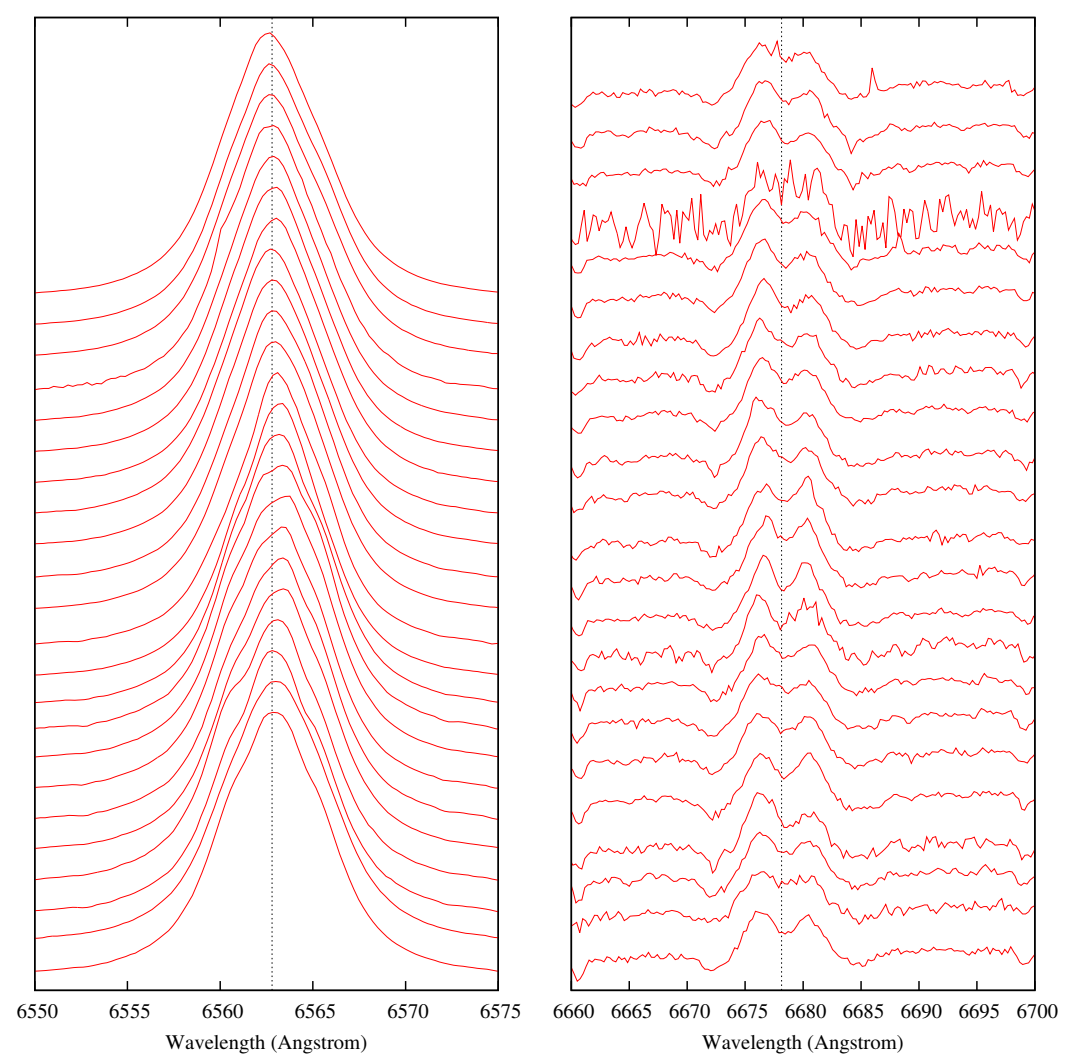

Figure 1: The normalized profiles of the $\mathrm{H} \alpha$ (left) and $\mathrm{He} \mathrm{I}$ (right) lines around the 2014 periastron passage. The lines are ordered from bottom to top from earliest to latest. The line profiles of the observations taken $\sim 27$ and $\sim 33$ days after periastron are not included in this plot since instrumental issues artificially widened the line. The vertical dotted lines show the rest wavelengths.

\section{Results}

\section{1 $\mathrm{H} \alpha$ line}

The $\mathrm{H} \alpha$ line remains single peaked through all observations, but shows a distinct asymmetry, as often seen in binary systems (e.g. Hanuschik et al. [[13]). This is interpreted as the the blending of the double (or multiple) components originating from the circumstellar disc (Fig. 1). The variation in the 2014 equivalent width (blue circles) is compared to the 2010/2011 observations (red plusses) in Fig. 2. The equivalent width (EW) is lower than previously detected, though it does follow the same trend. The peak occurs approximately 13 days after periastron, and appears to be decreasing by 19 days after periastron. The EW returns to the pre-periastron levels after 60 days from periastron. There is one other notable increase around 17 days before periastron, which is around the time of the first disc crossing. 


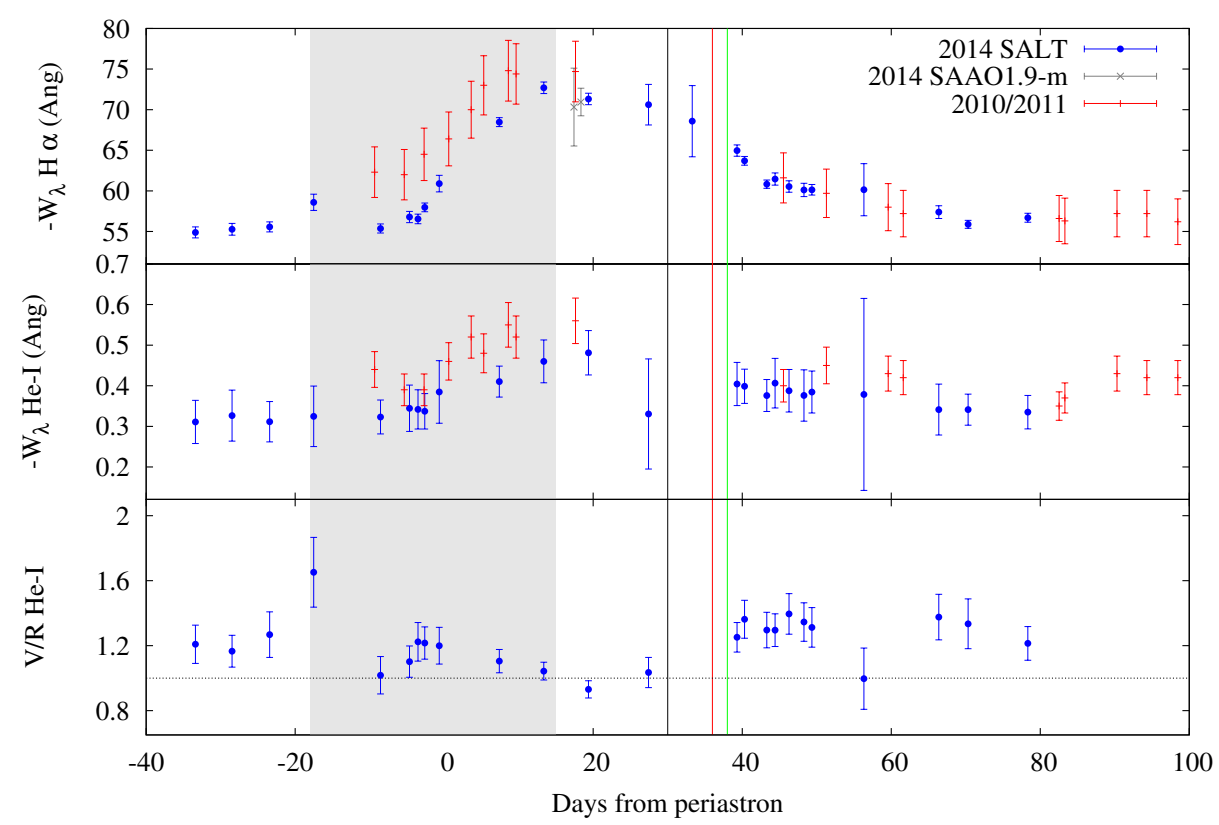

Figure 2: Equivalent width of the $\mathrm{H} \alpha$ (top), and equivalent width and V/R variation of the He I (middle and bottom) lines as observed with SALT in 2014 (blue circles). Due to the low signal to noise, the He I line could not be measured for the observations taken $\sim 33$ days after periastron. The 2010/2011 observations (from Chernyakova et al. [[]]) are shown for comparison. The black vertical line shows the time of the onset of the Fermi flare, while the red and green lines show the peak of the flare in 2010/2011 and 2014 respectively [ [5]. The grey shaded region indicates the period of the pulsar eclipse.

\subsection{He I line}

The He I (6678 $⿱$ A) appears double peaked in all observations and shows a varying asymmetry, with a generally stronger violet component. The trend in the EW width is similar to that previously reported (Fig. 2). The $V / R$ variation of the He I is measured by the height of the Gaussian line profile relative to the continuum, with the error determined from the statistical error of the fit. There is a clear increase in the asymmetry around the first disc crossing, a general decrease in $V / R$ after periastron, with the Red component becoming slightly stronger around the second disc crossing. The large change in $V / R$ at 56 days after periastron is believed to be due to the lower signal to noise associated with that observation, which is seen as the noisy He I profile in Fig. 1.

\section{Discussion \& Conclusion}

The general trend of the variation of the disc spectrum is similar to what was previously seen for PSR B1259-63/LS 2883 in 2010/2011. The H $\alpha$ EW width is consistently lower than the 2010/2011 observations until approximately 13 days after periastron. We suggest that this is due to the intrinsic variability associated with the Be star. The disc variability may be associated with the truncation and any asymmetry in the density of the disc introduced due to the interaction with the pulsar near periastron. Observations were limited near the onset of the Fermi flare, however, the variability shown could be consistent with a smooth variation in the disc as seen previously in, for example, $\delta$ Scorpii [प]4]. A comparison to multi-wavelength results around this period 
are presented in [15]. SALT is very well equipped to undertake long term synoptic monitoring of such binary systems since 100 per cent of observations are undertaken through queue scheduling operations.

\section{Acknowledgments}

Observations reported were obtained with the Southern African Large Telescope.

\section{References}

[1] Johnston S., Manchester R. N., Lyne A. G., Bailes M., Kaspi V. M., Qiao G., D’Amico N., PSR 1259-63 - A binary radio pulsar with a Be star companion, ApJ 387 (1992) L37

[2] Chernyakova M., Abdo A. A., Neronov A., et al., Multiwavelength observations of the binary system PSR B1259-63/LS 2883 around the 2010-2011 periastron passage, MNRAS 439 (2014) 432

[3] Abdo A. A., Ackermann M., Ajello M., et al., Discovery of High-energy Gamma-ray Emission from the Binary System PSR B1259-63/LS 2883 around Periastron with Fermi, ApJ 736 (2011) L11

[4] Tam P. H. T., Li K. L., Takata J., Okazaki A. T., Hui C. Y., Kong A. K. H., High-energy Observations of PSR B1259-63/LS 2883 through the 2014 Periastron Passage: Connecting X-Rays to the GeV Flare, ApJ 798 (2015) L26

[5] G.A. Caliandro, C.C.T. Cheung, J. Li, D.F. Torres, K. Wood, High energy $\gamma$-ray emission from PSR B1259-63 during 2014 and 2010 periastron passages, Conference proceeding. Swift: 10 Years of Discovery, 2-5 December 2014, La Sapienza University, Rome, Italy arXiv:1504.06343 (2015)

[6] Dubus G., Cerutti B., What caused the GeV flare of PSR B1259-63?, A\&A 557 (2013) A127

[7] Khangulyan D., Aharonian F. A., Bogovalov S. V., Ribó M., Post-periastron Gamma-Ray Flare from PSR B1259-63/LS 2883 as a Result of Comptonization of the Cold Pulsar Wind, ApJ 752 (2012) L17

[8] Kong S. W., Cheng K. S., Huang Y. F., Modeling the Multiwavelength Light Curves of PSR B1259-63/LS 2883. II. The Effects of Anisotropic Pulsar Wind and Doppler Boosting, ApJ 753 (2012) 127

[9] Takata J., Okazaki A. T., Nagataki S., et al., Modeling High-energy Light curves of the PSR B1259-63/LS 2883 Binary Based on 3D SPH Simulations, ApJ 750 (2012) 70

[10] Mochol I., Kirk J. G., Radiative Damping and Emission Signatures of Strong Superluminal Waves in Pulsar Winds, ApJ 776 (2013) 40

[11] Sushch I., Böttcher M., Pair cascades in the disk environment of the binary system PSR B1259-63/LS 2883, JHEAp 3 (2014) 18

[12] Vollmann K., Eversberg T., Remarks on statistical errors in equivalent widths, AN 327 (2006) 862

[13] Hanuschik R. W., Kozok J. R., Kaiser D., High-resolution emission-line spectroscopy of Be stars. III Balmer line profiles, A\&A 189 (1988) 147

[14] Miroshnichenko A. S., Fabregat J., Bjorkman K. S., et al., Spectroscopic observations of the $\delta$ Scorpii binary during its recent periastron passage, A\&A 377 (2001) 485

[15] Chernyakova M. et al., Multi-wavelength Observations of the Binary System PSR B1259-63/LS 2883 around the 2014 Periastron Passage, MNRAS 454 (2015) 1358 